\title{
Una técnica de las ciencias sociales: Escritura y auto observación en la investigación en artes
}

Guadalupe Arqueros ${ }^{1}$

gimenarqueros@yahoo.com.ar

\section{Resumen}

¿Cuáles son los sentidos de la investigación cuando es artística? ¿Cómo resolver la narración de la producción? ¿Es narrable el proceso creativo? ¿Qué significa textualizar? ¿Podrían las metodologías de investigación de las ciencias sociales aportar y vincularse con la textualidad del proceso? Con que técnica específica podría resolverse esta narración?

En la creación artística, la función de la escritura (memoria reflexiva) es considerada un instrumento central para la toma de distancia y contemplación, a la vez que la observancia del estilo posibilita leer el resonar de la obra, como una reconstrucción desde palabras de un proceso que es el de la creación misma.

La reelaboración del proceso a través de su escritura, constituye un esfuerzo consciente y guiado a la manera de una auto observación que completa el sentido de un recorrido y presenta otra especificidad en el aporte final del trabajo. La siguiente reflexión busca establecer un vínculo teórico entre las características de la técnica de la auto observación y la explicitación del proceso creativo a través de la escritura en la investigación en artes. Para ello retomamos una experiencia particular de formación en investigación de un Ciclo de Articulación en una Carrera de Artes de una facultad pública, donde el trabajo final incluye la posibilidad de una producción artística conjunta con una narración del proceso creativo. Consideramos que los matices de la auto observación como técnica, aportan a la escritura en artes (textualidad del proceso creativo) y dotan de una dimensión resonante socialmente, a las obras y al trabajo de quienes las producen.

Palabras clave: proceso, auto observación, creación, escritura.

\section{Abstract}

What are the meanings of artistic research? How to solve the narrative of production? Could research methodologies of social sciences contribute and connect with that textuality of the process?

1 Lic.en Filosofía, profesora de enseñanza superior y de enseñanza media, Especialista y maestranda en Metodología de la Investigación científica NEDIM.IIGHI-CONICET/UNNE. 
In creation, the function of writing (reflective memory) is considered a main instrument for taking distance and contemplation, while adherence to style it facilitates reading the resound of artwork, as a reconstruction from words of a process which is the creation itself. Despite the fact that who research isn't researching him or herself, writing constitutes a guided and conscious effort after a self-observation which completes the meaning of a journey, and it makes another contribution quality.

The following text seeks to establish a theoretical link between the features of methodological technique of self-observation and the explanation of creative process through writing at research in Arts.

Keywords: process, method, self observation, creation, writing.

\section{Introducción}

La discusión sobre el progreso en las artes tiene nuevas traducciones concentradas en interrogantes tales como: ¿En qué sentidos se habla de investigación artística? ¿Cuáles son los elementos para considerar que el trabajo ha expandido las fronteras de representación o aportó variables de construcción diferentes? Algunos autores consideran que el desarrollo de las artes visuales (como paradigmáticas) no viene dado por la investigación de datos contextuales (historia de los períodos artísticos, semiótica de las imágenes) sino por la producción de obra y las innovaciones que en este campo puedan aportarse. El arte sería un espacio donde no existe progreso acumulativo al modo científico, sino que su devenir se asemeja a saltos cualitativos diferenciados, no rigurosamente predecibles ni lineales. Consecuentemente la tarea de la investigación no parece aplicable sin más en el campo artístico, como tampoco los conceptos de la epistemología científica.

Distinguimos para continuar, la investigación sobre el arte, de la investigación artística propiamente dicha, reservando para esta última los sentidos de un tipo de indagación estética creativa en la constitución de nuevos lenguajes y obras. La investigación sobre arte seria la indagación teórica regular donde los objetos son obras, producciones, etc. o tópicos vinculados al mundo del arte. En la actividad de creación la función de la escritura como reelaboración del proceso y traducción a una textualidad, es considerada un instrumento central para la toma de distancia y en el ejercicio del estilo de la misma, resuena el hacer de la obra (Arias, 2010, p. 7). Pese a que quien investiga no está investigándose a si mismo se trata de un esfuerzo plenamente autoconsciente y guiado a la manera de una auto-observación. Es decir que el trabajo no finaliza con la producción, sino con la narrativa del proceso que la originó y completa su sentido en un recorrido experimental que es científico y que constituye un aporte diferente y enriquecedor. Para otros autores la escritura colabora en establecer el puente instrumental que aúna práctica artística y reflexión como un proceso y un resultado (Durán Castro, 2011, p.12), es decir que tiene un sentido no complementario sino constitutivo en la creación.

El presente texto indaga sobre un vínculo entre la técnica de la auto observación, proporcionada por la metodología de las ciencias sociales, y la explicitación de la instancia creativa a través de la escritura en la investigación en artes. Recorreremos la idea con 
Guadalupe Arqueros / Una técnica de las ciencias sociales: Escritura y auto [...]/57 un caso que analiza los requerimientos del Reglamento de Tesinas de una carrera de la Universidad Nacional (UNNE) que otorga títulos de Licenciados en Artes. Luego desarrollamos apuntes e ideas propias sobre la auto observación como técnica proveniente de las ciencias sociales, enriquece y contribuye cualitativamente al proceso creativo.

\section{Tesinas de la licenciatura en artes}

Como especialista en metodología y profesora en una Facultad de Artes de Universidad Nacional me encuentro integrando una Comisión de Tesina encargada de evaluar proyectos finales de la carrera de Licenciatura en Artes de la Facultad de Artes, Diseño y Ciencias de la Cultura de la Universidad Nacional del Nordeste con sede en la ciudad de Resistencia, ${ }^{2}$ Provincia del Chaco, República Argentina. Esta institución inicia sus actividades ofreciendo un Ciclo de Articulación para profesionales que posean un título de nivel terciario o universitario.

Los llamados ciclos de articulación son carreras que no tienen un dictado regular y continuo como las tradicionales de una unidad académica, sino que se cursan por única vez y por lo general presentan contenidos y ejes curriculares comprimidos. Son trayectos especiales que complementan la formación de profesionales dedicados al trabajo en el área hace tiempo. En su mayoría ofrecen títulos de investigación, como la licenciatura que analizamos, pero no de posgrado y sus bacantes son cubiertas por adultos que simultáneamente, en sus trayectos vitales, están trabajando. El ciclo educativo de tres años comenzó como carrera a término, otorgando certificaciones de Licenciatura en Artes, en tres ramas: visuales, musicales y escénicas. El dictado de asignaturas allí y el trabajo junto a la directora de la articulación en la Comisión de Tesinas, fue el motor de la reflexión del presente artículo.

Según el reglamento aprobado en el Ciclo de Articulación, las tesinas de finalización de carrera podrían tener dos modalidades; de investigación teórica, en este caso serían trabajos individuales; o bien de producción artística (teórico-práctica) trabajos grupales que además van acompañados de una memoria del proceso. El Reglamento de tesinas de la facultad explicita para las tesinas individuales:

Investigación: su formato seguirá los lineamientos de la investigación básica. Consistirá en un trabajo individual que refleje las capacidades teórico-críticas logradas por el alumno a lo largo de la carrera. El tema elegido por el alumno deberá referirse a aspectos que hagan a la teoría, historia, crítica, técnica o aspectos relacionados que constituyan una profundización y un aporte original e inédito en el campo en el que se inserta. (Res. $\mathrm{N}^{\circ} 016 / 12$ D. del 23 de febrero de 2012) ${ }^{3}$

Resaltamos tres detalles importantes en la extracción previa:

a) la limitación en la modalidad individual del trabajo, que descarta una construcción colectiva del conocimiento que resulta como aporte;

2 F.A.D. y C.C./UNNE. http://www.artes.unne.edu.ar/ y http://www.artes.unne.edu.ar/artes-combinadas.html [consulta: 25-7-2015]

3 Resolución de la Facultad de Artes, Diseño y Ciencias de la Cultura, de la Universidad Nacional del Nordeste, Resistencia Chaco, Argentina. Anexo: Reglamento de tesina. 
58/ Perspectivas Metodológicas /19/Vol. II /Año 2017

b) el concepto teórico de investigación básica, sin desarrollar sus matices epistémicos, aplicado al campo de la teoría sobre artes,

c) el reglamento muestra conocimiento prediciendo las orientaciones de los trabajos, en consonancia con lo explicado por los autores citados.

Cuando los cursantes optan por la investigación teórica coincidimos con el análisis de Tolosa para el caso de España: las tres líneas de investigación universitaria se agrupan en confluencias interdisciplinares por un lado, trabajos históricos por otro y temas específicos (Tolosa, 1998, p. 64). Las investigaciones de tipo teóricas (sobre artes) representan las elecciones mayoritarias de los futuros graduados, concentrándose en tópicos de historia y educación artística. Las preferencias resultan ser la teoría estética, historia del arte, crítica y materiales e instrumentos. Elaboramos un gráfico [Figura 1] que puntualiza los contenidos hallados en las tesinas individuales con orientación teórica:

Figura 1: Recorridos temáticos de las tesinas del Ciclo de Articulación en Artes de la UNNE. (Datos de la Comisión de Tesina, al mes de marzo de 2015)

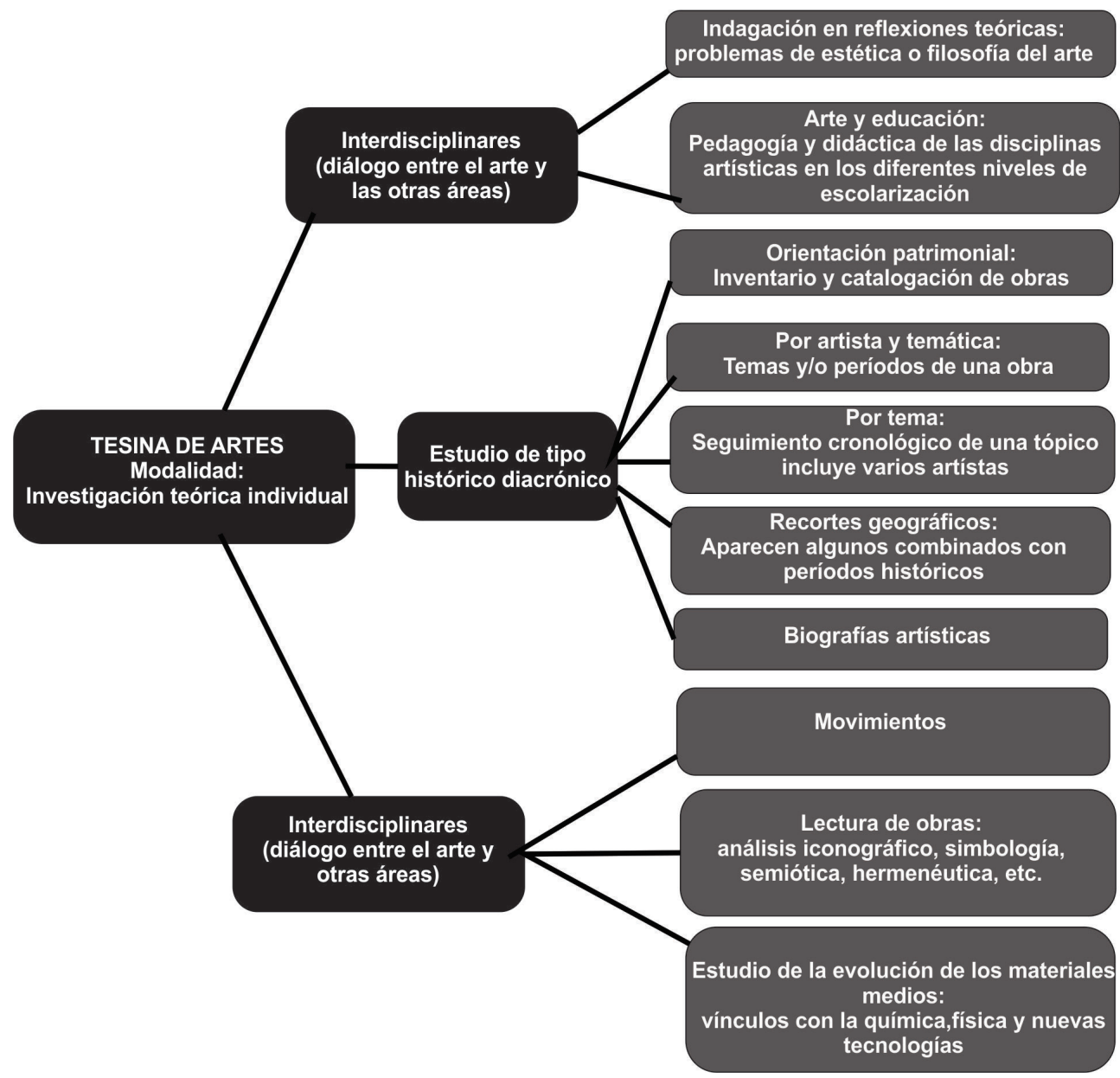

En las investigaciones teóricas se destacan en especial los estudios históricos, referidos a la historia del arte y artistas, locales y regionales en especial. También encontramos que 
Guadalupe Arqueros / Una técnica de las ciencias sociales: Escritura y auto [...]/59 los tópicos se solapan pese a nuestro afán analítico, por ejemplo en las biografías, donde aparecen estudios pormenorizados de un recorte de obras o en la confluencias disciplinares específicas y el desarrollo de una ciencia (como en la química y los análisis de los materiales). También hay conjunción en los recortes históricos y geográficos (aparecen títulos como el arte de la región en el periodo... por ejemplo).

\section{Egresar con una producción artística}

En las tesinas de realización (llamadas teórico-prácticas según el reglamento) a la hora de acompañar desde la dirección de las mismas, nos encontramos con la dificultad de dilucidar: ¿Qué tipo de saber específico otorga a un creador o productor un grado académico de investigación, como supone el de licenciado? ¿Qué pide el reglamento para la obtención del título en el caso de esta modalidad de tesina? ¿Qué género de escritura y que metodología acompaña la producción, traducida a texto? ¿Es posible describir un proceso creativo?

Si tomamos la letra, nos dice para la realización de una obra como producción artística:

a) en el campo de las artes visuales: se permitirá la presentación de los resultados de una obra o serie con la explicitación escrita que partirá de una reflexión teórica sobre la creación de la misma, investigación del tema, objetivos y público al que va destinada, y que dé cuenta de los procedimientos técnico/procedimentales aplicados.

b) en el campo de las artes escénicas: podrá consistir en una obra dramática original que deberá ser acompañada por una memoria en la que se desarrolle la metodología de trabajo, la problemática abordada y los recursos artísticos y técnicos utilizados en su producción.

c) en el campo de las artes musicales: [...] una Memoria que contenga los elementos estéticos y conceptuales como también tecnológicos utilizados. ${ }^{4}$

Analizando los requerimientos del reglamento para los egresos con producción hallamos una restricción al trabajo individual. Para la obtención del título la producción debe ser grupal, la introspección será entonces grupal. El reglamento estipula que los trabajos teóricos sean hechos por un solo estudiante y los de producción sean colectivos, sin embargo no prevé una actividad artística individual y se muestra condicionante en este sentido. La producción sería un tipo de trabajo de experimentación con nuevos lenguajes junto con el complemento de un escrito que continúa la obra original.

Con respecto al texto que debe acompañar las obras, es denominado explicitación escrita y reflexión teórica sobre la creación, para las artes visuales; memoria para las escénicas y musicales y se refiere a un trabajo usualmente de no más de quince carillas donde se describe el proceso, los antecedentes, las técnicas, el nivel de innovación, etc., que 4 Op. cit., p 1. 


\section{0/ Perspectivas Metodológicas /19/Vol. II /Año 2017}

trae aparejada la obra que se presenta. Este pedido de manifiesto resulta un requisito al menos interesante para aquellos que sean también creadores y que culminando el proceso de formación deben realizar una explicitación autoconsciente y porque no una auto observación, del proceso mismo. En el reglamento sin embargo falta especificidad sobre la composición del escrito y es en el caso de los licenciados en artes visuales donde únicamente aparece una reflexión teórica sobre la creación, ya que en las restantes ramas solo se solicita metodología de trabajo, problemática abordada y recursos artísticos y técnicos utilizados para las artes escénicas y un escueto elementos estéticos y conceptuales como también tecnológicos utilizados para los licenciados en artes musicales.

Las mayores dificultades se presentan cuando hay que destacar la producción como un trabajo original, en vinculación con los antecedentes en el campo y cuando hay que observar-describir un proceso creativo grupal, operación que hemos llamado arbitrariamente textualizar. Tal vez sea de ayuda en este punto recordar que para referirse a la instancia que aúna investigación y producción desde la Universidad de Granada Marín Viadel utiliza el término IENA (Investigación en Artes) en las llamadas tesis prácticas (con obra original) estipulando sus características. (Marín Viadel, 1998, p. 87)

\section{Investigación artística y académica}

Expedir un título de licenciatura es para la institución superior otorgar una certificación inicial en investigación, por eso es fundamental establecer las posibles modalidades cada vez que se menciona la investigación en artes. Tomando arbitrariamente como paradigma las artes visuales el desarrollo de las mismas, como actividad cultural humana no viene dado a través de la investigación de datos contextuales (historia de los períodos, semiótica de las imágenes, etc., que sí corresponderían a la investigación teórica) sino por la producción de obra y las innovaciones que en este campo puedan aportarse.

La confusión surge cuando se asume que la producción sin más es una tarea de investigación. En un artículo clásico escribe Tolosa al respecto:

$\mathrm{Al}$ estatus que puede adquirirse como artista, legitimado por el campo del arte, viene a añadirse el de investigador en Arte legitimado por la Universidad. Las cualidades que se necesitan para obtener estas dos legitimaciones, son complementarias. Se trataría de saber cómo pueden interaccionarse o, incluso, superponerse. De qué manera esta noción de investigación en Arte, asumida por los criterios de investigación en la Universidad, plantea de forma inédita, una cierta aproximación a la práctica del arte y su explicitación. (Tolosa, 1998, p. 59)

Las carreras que articulan estas trayectorias se enriquecen con la presencia de artistas y productores de obras entre sus estudiantes y sus programas por lo general, aprovechan esta instancia, brindando un tipo de especulación teórica y sobre la práctica misma. Esta reflexión es diferente en calidad y profundidad a la recibida en los centros de formación docente, de donde egresaron en general los inscriptos. Por otro lado Tolosa menciona como la investigación en artes puede echar luz sobre los procesos más internos de la crea- 
Guadalupe Arqueros / Una técnica de las ciencias sociales: Escritura y auto [...]/61 ción. Nos preguntamos entonces ¿¿Cuál es el instrumento como conjunto de pasos, o la técnica que pueda ser usada y enseñada para este vínculo, para textualizar este proceso?

Avanzando, conviene deslindar dimensiones. Borgdorff (2004) diferencia los planteos hacia adentro de la investigación en artes y clasifica tres enfoques. Los inclinados a la ontología resultan en visiones sobre la naturaleza de los objetos de investigación. Abarcarían desde los comienzos de la reflexión estética, cuando con Platón el artista copiaba los eidos primeros del topos uranos. Hasta las modernas teorías de la ficción, en literatura, por ejemplo y los contratos con lectores (Pozuelo Yvancos, 1993). La escala metafísica de los objetos producidos por el arte discuten los parámetros de una realidad intersubjetivamente compartida. Además el arte contemporáneo en especial, se muestra esquivo y plagado de ambigüedades que imposibilitan un análisis esencialista y esquemático (Oliveras, 2009). En el grafico [Figura 1] las indagaciones ontológicas se corresponden con las filosóficas y estéticas propiamente dichas (reflexiones teóricas).

Las cuestiones epistemológicas sin embargo versarían sobre el tipo de conocimiento discursivo que se produce y sus vínculos con otros campos del saber humano. Si bien el análisis completo es de carácter epistémico, las observaciones se centran en el alcance y la envergadura del contenido producido, como conocimiento sobre los campos del arte analizados.

Y por último los métodos adecuados y las técnicas seleccionadas y sus relaciones con las ciencias naturales, las humanidades y en especial las ciencias sociales y la historia. Estos últimos se llamarían análisis metodológicos. Como los puntos de contacto son diferentes, las técnicas y métodos también lo serán. En el ejemplo que analizamos de las tesinas de la Carrera de Articulación, prevalece la metodología historiográfica ya que hay búsqueda heurística y de fuentes sobre biografías de artistas y trayectos profesionales que se corresponden en especial con los recorridos históricos y diacrónicos. Sin embargo existe otro sector temático vinculado al estudio y evolución de materiales y medios que usan las técnicas de las ciencias naturales, por ejemplo la química y la física. ${ }^{5}$ En un cuadro ordenamos las posibilidades [Figura 2].

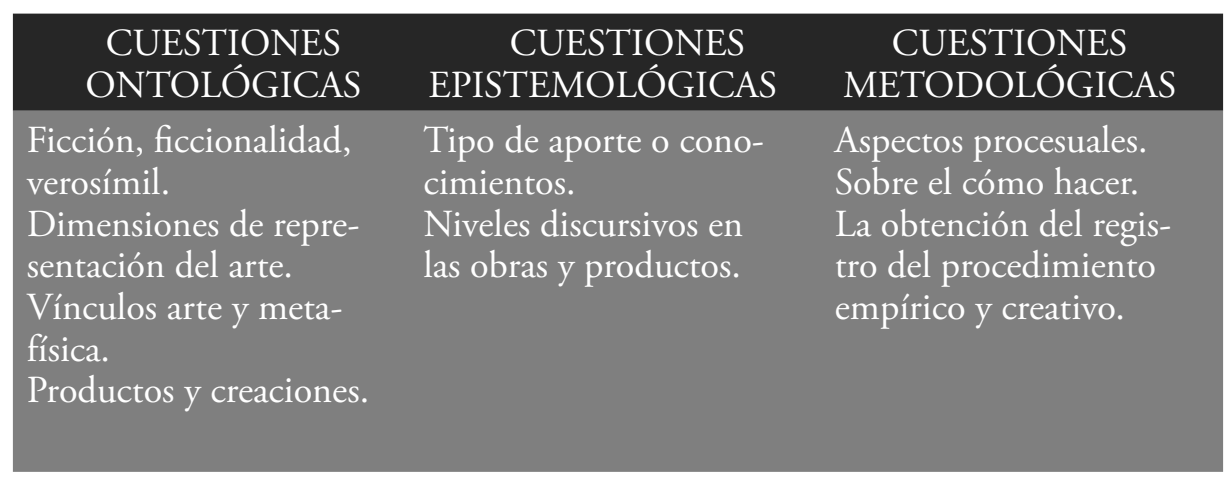

Figura 2: Clasificación de los principales aspectos según (Borgdorff, 2004, pp.15-18)

5 En este caso se habla también de una investigación para el arte. 


\section{2/ Perspectivas Metodológicas /19/Vol. II /Año 2017}

Hasta el momento se discutieron las tesinas teóricas, aquellas donde la investigación es sobre objetos vinculados al mundo del arte. Pero el nudo comienza cuando aparece la urgencia de la memoria descriptiva de las tesinas de producción: ¿¿Cuál es el género de esta narración? ¿Qué le recomendamos al grupo cuando tiene que narrar compulsivamente su proceso para la obtención del título? Dirimiendo los puntos de vista de la discusión y contraponiéndose a quienes impugnan esta modalidad de egreso, Marin Viadel considera que:

[El rechazo a las tesis prácticas] lo que discute es la conveniencia de que el dibujo, la pintura, la escultura, la fotografía o el diseño, sean un campo universitario. De otro modo no encuentro qué pueda ser una aportación original al dibujo que no sea el dibujo. Lo que rige en arte son las obras de arte. El método iconológico no es una aportación a la pintura sino a la disciplina de la historia del arte. Para desarrollar la pintura no hay más remedio que pintar buenos cuadros. Este es el objetivo fundamental de las tesis prácticas. (Marin Viadel, 1998, p. 91)

Este punto de vista puede sonar radical si no se comprende que el autor piensa en las grandes revoluciones estéticas principalmente pero no solamente, también científicas, que modificaron y hasta multiplicaron las subjetividades y formas de ver el arte como manifestación humana, diversificando la sensibilidad de una realidad nunca única.

\section{Auto observación}

En cualquier proceso creativo hay un pensamiento reflexivo, que no coincide con el mitema de la inspiración o en su extremo la habilidad mecanizada. Es el momento del tema, el asunto, los materiales, las decisiones expresivas y de comunicación en la obra, los posibles efectos en los espectadores, etc. (Duran Castro, 2011, p. 7) Frente a la posibilidad de que un texto refleje la lógica misma de este tipo de proceso Arias va más allá, pregunta y contesta:

¿Cómo tomar distancia de los procesos de creación y reflexión teórica de las obras para delimitarlas como objetos de investigación? Dicha distancia es posible en la escritura, no simplemente como elaboración teórica sino como ejercicio de un estilo en el que:

[...] resuena el hacer de la obra. [...] el objetivo de toda investigación en artes, es ante todo construir ese método comprendido no en el sentido tradicional de un sistema de pasos a seguir con el fin de ampliar el ámbito del conocimiento, sino como la construcción de una dinámica en la que se hagan posibles diversas replicas en las que el movimiento creativo encuentre cierta distancia sobre sí mismo. (Arias, 2011, pp. 7-8)

Pese a no coincidir con el subsiguiente posicionamiento que Arias deriva de las precedentes afirmaciones, cuando considera que a través del texto se unifica una separación que supone entre teoría y práctica; en algo si coincidimos y es que la escritura posibilitaría hacer explícito un sentir de quien crea, y que como recurso de auto observación puede conducir a una teoría o a la conceptualización del proceso. ${ }^{6}$

6 Tomando el término teoría como lo entendía Ladriere de modelización de un tipo de realidad que 
Guadalupe Arqueros / Una técnica de las ciencias sociales: Escritura y auto [...]/63

Habría variables frecuentes (en un sentido no específico) en el proceso creativo de los individuos, que pueden rastrearse, serian estas las réplicas mencionadas en la cita. Se justifica de esta manera el registro escrito en código de auto observación, buscando líneas comunes y desalojando definitivamente la estética kantiana del genio creativo único, falo-logocéntrico retomada por la teoría especulativa del arte. Aunque al decir de Tolosa: "Existen también sectores que cultivan el mito del puro creador, que no necesita ejercitar su modestia, ni contrastar su obra de una manera crítica" (Tolosa, 1998, p. 61).

Si tomamos a Delgado y Gutiérrez, (1999, p. 165) discutiendo la auto observación como un tipo de metodología que refleja y propicia el cambio social conversacional, veremos que hay una serie de rasgos que podrían ser aplicables a la escritura del proceso creativo como exigen las memorias académicas. Analizando sus rasgos hay que delimitar que observador y observado son posiciones y no personas o compartimentos inamovibles, el término con el que se denominarán será sistema observador y sistema observado y sucederán en el mismo individuo. En este marco la auto observación es una forma derivada de la relación nosotros en orientación otro, es decir es una reflexividad con base en dicha orientación, (Delgado y Gutiérrez, 1999, p. 163).

En la investigación empírica tradicional (pensamos en la sociología cualitativa) el sistema observado es objeto de observación externa directa o indirecta con registros cuantitativos y cualitativos. Pero en la auto observación tal como la sugerimos para este campo, el sistema observador se escinde en estado observador y estado observado. Remarcan los autores para las ciencias sociales, la necesaria prioridad en los roles, de tal manera de que la configuración sea actor - observador y no a la inversa (¿podríamos traducir en creador-observador?).

En la auto observación se da el proceso inverso al de la observación participante, donde se aprende a ser nativo de una cultura extraña; aquí sin embargo se extraña de la propia cultura o del proceso tomando distancia y ordenando. Partiendo de la noción de sistema observador y sistema observado, los autores recuerdan que la necesaria prioridad la tiene el orden que desdobla el sistema en actor y luego observador. Esto nativo próximo inundado (Flick 2004, p. 176) con orientación otro (¿sí mismo?) se convierte en constructor y autor del mundo de percepción lo que hace de la metodología una arqueología vivencial, al decir de Delgado y Gutierrez (1999, p. 168). También es fundamental que el observador sea un nativo próximo y no un nativo remoto, lo que implica que el sujeto debe abandonar, o debe haber dejado su condición de participante, para constituirse en estado observador del sistema y este movimiento de giro el que trae la distancia autoreflexiva.

La posición auto observadora es dependiente de una teoría de la fractalidad social en la medida en que es relacional y existen núcleos de complejidades comunes en los niveles y escalas de observación. La hipótesis de base radica en que no es el individuo aislado quien piensa e interpreta, sino que es siempre producido y reproductor de esas instituciones y discursos de tipo abstracto. En este sentido la metodología cualitativa entiende el sistema como una unidad holística, donde tanto quien investiga como los sujetos se encuentran atravesados por los rasgos de cultura y educación que son los del uso de su comunidad. ${ }^{7} \mathrm{Y}$ estos rasgos componen la complejidad de la interpretación misma.

se está analizando, y no en el sentido rígido omniexplicativo que conserva para la ciencia positiva (Ladriere, 1977)

7 Recordamos una frase importante de la historia de la sociología que puede ejemplificar el caso: La 
Frente al desboblamiento en sistemas de observación, resulta pertinente aquí una cita de Hammersley y Atkinson referida al método etnográfico:

El etnógrafo en su dinámica de inmersión y distanciamiento simultáneos, puede vivir una especie de esquizofrenia. Pero ese sentimiento, $\mathrm{u}$ otros equivalentes, deberían ser tomado por lo que realmente es. No es algo que necesariamente tenga que evitarse o ser sustituido por sensaciones más agradables de bienestar. (Hammersley y Atkinson 1994, p. 118)

Escribir es reconstruir el mundo a través de la experiencia del sujeto y usufructuar de la fractalidad social, análoga y no equivalente en cada uno, que aúna complejidades comunes ${ }^{8}$. El núcleo entonces es que en la memoria explicativa de los trabajos que dan cuenta de las producciones artísticas, asoma un tipo de narración que sobrepasa la dinámica de la creación individual. En la memoria se cruzan los tipos diferentes de enfoque que puntualizara Borgdorff (2004). Conocer es entonces tener experiencia de y experiencia en, más la conceptualización obligatoria del proceso que lo convierte en transferible, a la vez que le quita idealización romántica y elitismo falo-logocéntrico a los creadores. Esta visión se impregna más con las presentaciones emergentes del arte contemporáneo que se autodefine como desdefinido.

La posibilidad de enseñar la narratividad y la distancia del propio proceso creativo en alumnos y estudiantes adultos de artes que conllevan sus propias experiencias vitales de artistas, implicó la adaptación de una técnica para la recreación de un proceso y también un aporte al conocimiento de la educación en artes.

El dilema seguirá siendo ¿Cuánto tarda el proceso y si es posible transformar artistas y docentes en investigadores?

\section{Bibliografía}

- Arias, J. C. (2010). "La investigación en artes: el problema de la escritura y el método”. Cuadernos de Música, Artes Visuales y Artes Escénicas, 5(2), pp. 5-8.

- Arqueros, G. (2014). "Explicitar el proceso. Auto-observación y escritura en la investigación en artes”. En Actas I Post Congreso ICQI, I Congreso de Investigación Cualitativa en Ciencias Sociales. IIQI, CIECS CONICET/UNC. Universidad Siglo 21. Recuperado de http://www.21.edu.ar/investigacion-ponencias-congreso-investigacion-cualitativa-ciencias-sociales.html

— Borgdorff, H. (2007). El debate sobre la investigación en las artes. Recuperado de: http://gabrielabarrionuevo.com.ar/lenguajeeinvesticacion/el-debate-sobre-la-investigacion-en-las-artes/.

historia del individuo, no es más que una especie de concreción de la historia colectiva de su grupo o clase social. Bourdieu, Pierre. Esbozo de una teoría de la práctica. 1977.

8 Aquí vendría a coincidir la llamada tarea política asignada por Feliu y Jaunesse (Feliu y Jaunesse 2007) a la autoetnografía contemporánea. El descentramiento de quien escribe hecha raíces en lo particular e intransferible del proceso creativo, pero también vincula posibilidades de expandir y si no reproducir, reconocer uniformidades que des-hegemonizan el genio falo-logocéntrico. 
Guadalupe Arqueros / Una técnica de las ciencias sociales: Escritura y auto [...]/65

— Delgado, J. M. y Gutierrez, J. (1999). "Teoría de la observación”. En Métodos y técnicas cualitativas de investigación en ciencias sociales. Madrid: Síntesis.

— Della Porta, D. y Keating, M. (Eds.) (2013). Enfoques y metodologías de las ciencias sociales. Una perspectiva pluralista. Madrid: AKAL.

- Duran Castro, M. (2011). "La escritura en las disciplinas artísticas". Cuadernos de Música, Artes Visuales y Artes Escénicas, 6(2), pp. 5-12.

— Feliu, J. y Lajenusse, S. (2007). "Nuevas formas literarias para las ciencias sociales: el caso de la autoetnografía". Athenea Digital, (12), pp. 262-271.

— Fernández Arenas, J. (1982). Teoría y metodología de la historia del arte. Barcelona.: Antrophos.

— Gallart, M. A. (1992). La integración de métodos y la metodología cualitativa. Una reflexión desde la práctica de la investigación. En Vasilachis de Gialdino, I. y otros, Métodos cualitativos I y II (pp. 107-119). Buenos Aires: Centro Editor de América Latina, (pp. 107-119).

- Hammserley, M. y Atkinson, P. (1994). Etnografía. Métodos de investigación. Buenos Aires: Paidós.

— Ladrier, J. (1997). El reto de la racionalidad. Salamanca: Sígueme.

- Marin Viadel, R. (1998). El espacio de la 'IENA': ¿Qué es la investigación en arte y cuál es su función entre las investigaciones de bellas artes? En La investigación en bellas artes. Tres aproximaciones a un debate. Granada: Grupo Editorial Universitario.

- Mendioca, G. (2003). Sobre tesis y tesistas. Lecciones de enseñanza-aprendizaje. Buenos Aires: Espacio.

- Montoya López, A. (2006). "La investigación en arte. Como acceder a nuevas formas de expresión”. Artes. La Revista, 12(6).

- Oliveras, E. (2009). La metáfora en el arte. Retórica y filosofía de la imagen. Buenos Aires: Emecé.

— Pozuelo Yvancos, J. M. (1993). Poética de la ficción. Madrid: Síntesis.

- Rodríguez Gómez, G., Gil Flores, J. y García Jiménez, E. (1999). Metodología de la investigación cualitativa. Málaga: Aljibe.

— Tolosa, J. L. (1998). "Modelos de investigación en bellas artes". En La investigación en bellas artes. Tres aproximaciones a un debate. Granada: Grupo Editorial Universitario. 